\title{
Associations between clinical data and computed tomography features in patients with epidermal growth factor receptor mutations in lung adenocarcinoma
}

\author{
Yiyuan $\mathrm{Cao}^{1} \cdot$ Haibo $\mathrm{Xu}^{1} \mathbb{D} \cdot$ Meiyan $\mathrm{Liao}^{1} \cdot$ Yanjuan $\mathrm{Qu}^{1} \cdot$ Liying $\mathrm{Xu}^{1}$. \\ Dongyong $\mathrm{Zhu}^{1} \cdot$ Bicheng $\mathrm{Wang}^{2} \cdot$ Sufang Tian $^{2}$
}

Received: 15 June 2017 / Accepted: 22 September 2017 / Published online: 7 October 2017

(C) The Author(s) 2017. This article is an open access publication

\begin{abstract}
Background To analyse the differences in computed tomography (CT) features between patients with lung adenocarcinoma who have epidermal growth factor receptor (EGFR) mutations and those who have wild-type EGFR.

Methods Patients with lung adenocarcinoma $(n=156)$ were enrolled from October 2013 to March 2016, including 56 patients with wild-type EGFR and 100 patients with EGFR mutations. Two independent radiologists evaluated patient characteristics and imaging features. Chi-squared test, Fisher's exact test or ANOVA was applied to discriminate clinical and CT characteristics between the genotypes. A prediction tool for EGFR mutation was devised from principal component analysis.

Results The proportion of females and non-smokers in the exon 19 deletion and exon 21 missense groups was higher than in the wild-type group $(P<0.01)$. Severe emphysema was higher in the wild-type group than in the exon 19 deletion group $(P<0.01)$. The maximum diameter in the mediastinal window $\left(\mathrm{MaxD}_{\text {mediastinal }}\right)$ in the wild-type group was longer than in the exon 19 deletion and exon 21 missense groups. The minimum diameter in the mediastinal window $\left(\mathrm{MinD}_{\text {mediastinal }}\right)$ in the wild-type group was also longer than in the exon 21 missense group, with a significant difference $(P<0.05)$. The tumor shadow disappearance rate (TDR) in the exon 19 deletion group was higher than in the wildtype group. Ground glass opacity (GGO) appeared to be
\end{abstract}

Haibo Xu

haiboxu1120@hotmail.com

1 Radiology Department, Zhongnan Hospital of Wuhan University, Wuhan University, Wuhan, China

2 Pathology Department, Zhongnan Hospital of Wuhan University, Wuhan University, Wuhan, China more common in the exon 19 deletion group $(P=0.010)$. The prediction score for exon 19 deletion mutation was: $0.305 \times$ gender $+0.254 \times$ smoking history $+0.198 \times$ MaxDmediastinal + TDR $\times 0.254+0.280 \times$ GGO $+0.095 \times$ emphy sema. The sensitivity and specificity for predicting exon 19 deletion were 59.09 and $76.79 \%$, respectively. The prediction score for the exon 21 missense mutation was: $0.354 \times$ gender $+0.291 \times$ smoking history $+0.410 \times$ MaxDmediastinal $+0.408 \times \mathrm{MinD}_{\text {mediastinal }}$. The sensitivity and specificity for predicting exon 21 missense mutation were 72.34 and $78.57 \%$, respectively.

Conclusion As well as gender, smoking history and GGO, adenocarcinomas with EGFR mutation were significantly associated with emphysema, TDR, and the diameter in the mediastinal window. As exon 19 deletion and 21 missense mutations might be predicted by those features, the scoring system might be valuable for clinical diagnosis.

Keywords Computed tomography - Epidermal growth factor receptor $\cdot$ Shadow disappearance rate $\cdot$ GGO .

Emphysema

\section{Introduction}

Lung and bronchus cancer is the leading cause of cancerrelated deaths worldwide, with an approximate 0.16 million deaths in the United States annually [1], and 0.61 million deaths per year in China [2]. Lung cancers are classified by histologic features as non-small cell lung cancer (NSCLC) and small cell lung cancer (SCLC). NSCLC accounts for 85-90\% of lung cancers. Most of these patients present with inoperable advanced or metastatic disease with an extremely poor prognosis. The development of molecular-targeted therapies has revolutionized NSCLC therapy by affording 
better tumor control and selectivity with less toxicity than traditional chemotherapy [3].

Epidermal growth factor receptor (EGFR) is a transmembrane receptor tyrosine kinase involved in the signaling pathways that regulate cell proliferation, apoptosis, angiogenesis, and invasion [4-6]. Ninety-five percent of EGFR mutations are found in adenocarcinomas, which are the most common histologic type of NSCLC [7]. EGFR mutations with exon 19 deletions and L858R point mutation in exon 21 occur most frequently in NSCLC, and have a high response rate of approximately $70 \%$ to EGFR tyrosine kinase inhibitor (TKI) therapy $[4,6,8,9]$. Exon 20 insertions are also well described and have been associated with TKI resistance $[10,11]$, representing 4-9\% of EGFR-mutant lung cancers [11-13]. Exon 20 point mutations like T790M are rarely identified in pretreatment NSCLC tumors [11, 13, 14], but can be found in $>50 \%$ of NSCLC tumors which have acquired resistance to TKI therapy $[11,14]$. Some uncommon point mutations such as exon 21 (at L861) and exon 18 (at G719) were found to have a response rate to EGFR-TKI therapy of approximately $50 \%[11,13]$. However, wild-type NSCLC has been reported to be less sensitive to TKI therapy [15].

It would be very useful with regard to treatment if the EGFR mutation status of NSCLC could be identified without molecular examination. To our knowledge, only a few studies have attempted to determine the relationship between imaging features and molecular findings. Computed tomography (CT) imaging plays an important role in diagnosis and response assessment in cases of NSCLC. Therefore, the purpose of this study was to retrospectively identify CT features that correlate with EGFR mutation status in lung adenocarcinomas in a cohort of East Asian patients.

\section{Materials and methods}

\section{Patient selection}

This retrospective study was approved by the institutional review board. Informed consent was waived. From October 2013 to March 2016, a search of the electronic medical records at Zhongnan Hospital of Wuhan University revealed that 385 patients had undergone EGFR mutation testing.

Exclusion criteria were (1) no preoperative CT on the Picture Archiving and Communication System (PACS, Neusoft Vision 3.1) including both non-contrast-enhanced CT and contrast-enhanced CT, (2) non-adenocarcinoma cell type, (3) cases with multiple lesions on CT which individually could not be conclusively correlated with the lesions documented in the pathology report, (4) difficult to contour the tumor margin on CT images, (5) incomplete electronic medical records, and (6) the specimens were not obtained from the lung resection or open lung biopsy. Therefore, 229 patients were excluded.

Clinical and pathological data collected for analysis included age, gender, stage, smoking status (nonsmoking defined as patients who had never smoked), and emphysema status (the severity of emphysema was graded according to Goddard's Grade [16] and categorized into two groups). Tumor staging was according to the American Joint Committee on Cancer Staging Manual, 7th edition [17].

\section{Histologic evaluation and molecular analysis}

All histological specimens were formalin-fixed and stained with hematoxylin-eosin as part of the routine regulations of our hospital. Two board-certified pathologists (BW with 18 years experience of pathologic diagnosis of lung cancer, and ST with 17 years experience of pathologic diagnosis of lung cancer) reviewed the pathologic specimens and recorded the pathologic type of each tumor according to the 2015 WHO Classification of Lung Tumors [18].

All cases were analyzed for EGFR mutations at exons 18-21 using a pyrosequencing assay based on polymerase chain reaction (PCR). The sequence analysis was performed using a real-time quantitative PCR system (Mx3000P; Stratagene, La Jolla, CA, USA).

All cases underwent EGFR mutation analysis. The specimens were formalin fixed and paraffin embedded. Genomic DNA was extracted from the tumor tissue and EGFR mutations in exons 18 (G719X), 19 (deletion), 20 (T790M, 20-Ins and S768I), and 21 (L858 and L861Q) were detected using a fluorescence PCR diagnostic kit (Amoy Diagnostics Co., Xiamen, China). All EGFR mutation analysis was performed in the pathological laboratory by a board-certified pathologist (ZX with 10 years experience of pathologic diagnosis of lung cancer). The intensity score was defined as-cycle threshold $(\mathrm{Ct})$ scores $0-26$ were considered strong positive expression, 26-29 were considered weak positive expression, and $>29$ were considered negative expression.

\section{CT imaging}

All patients included in the study had preoperative chest CT scans available, including both pre-contrast-enhanced and contrast-enhanced scans, which had been conducted within 1 month prior to surgery. CT imaging was performed using one of the 2 CT systems (Sensation 16 and Somatom Definition; Siemens Medical Systems, Erlangen, Germany). For the Sensation 16, the parameters were $120 \mathrm{kV}$ and $100 \mathrm{~mA}$, with dose modulation. Images were reconstructed with a section thickness and an interval of $5 \mathrm{~mm}$ without a gap using the B50 algorithm and with a section thickness of $1 \mathrm{~mm}$ using the B80 algorithm. For the Somatom Definition, the parameters were $120 \mathrm{kV}$ and 
100-400 mA, with dose modulation. Images were reconstructed the same as Sensation 16. Contrast-enhanced images were obtained after intravenous administration of iopromide with $300 \mathrm{mg}$ of iodine/mL (Ultravist 300; Bayer Pharma, Berlin, Germany) at a rate of $3.0 \mathrm{~mL} / \mathrm{s}$ using a high-pressure syringe, with a dose based on patient weight (70-120 mL). CT scanning was performed with a 45-s delay.

\section{CT interpretation}

All images were viewed at mediastinal (width $350 \mathrm{HU}$, level $50 \mathrm{HU}$ ) and lung window (width $1500 \mathrm{HU}$, level $-700 \mathrm{HU})$ settings for axial images on PACS. Two boardcertified thoracic radiologists interpreted the CT images. Both radiologists (YC and YQ had 9 and 19 years of experience, respectively, in chest image interpretations) were aware that the patients had lung cancer, but were blinded to the pathologic diagnosis as well as the EGFR status.

The imaging characteristics of the primary lesions were recorded. In terms of morphologic characteristics, we calculated the tumor size, TDR, and the relative enhancement (Erel). The presence or absence of calcification, air bronchograms, bubble-like lucency or cavities, vessel convergence sign, lobulation, pleural retraction, GGO, pneumonia-like consolidation, spiculation, pleural effusion and pericardial effusion was assessed. TDR $=1-\mathrm{MaxD}$ mediastinal $\times \operatorname{MinD}_{\text {mediastinal }} /\left(\operatorname{MaxD}_{\text {lung }} \times \operatorname{MinD}_{\text {Lung }}\right)$, where MaxD mediastinal and MinD mediastinal meant the length of the longest and shortest diameter in millimeters in the mediastinal window, and the $\mathrm{MaxD}_{\text {lung }}$ and $\mathrm{MinD}_{\text {Lung }}$ meant the diameter in the lung window [19]. Erel = (Apost-Apre $) /$ Eart, where Apost was contrast-enhanced CT attenuation of the lesion, Apre was unenhanced CT attenuation of the lesion and Eart was the attenuation of the aescending aorta in contrast-enhanced CT; these (regions of interest) ROIs were all in the same transverse section [20]. Lobulation was defined when a portion of the surface of a lesion showed a shallow wavy configuration (except for regions abutting the pleura) and was calculated by numbers. Pneumonia-like consolidation is described as a homogenous opacity lesion in the lung defined by little or no volume loss, effacement of blood vessel shadows, and sometimes by the presence of an air bronchogram, and no concomitant bacterial pneumonia or obstructive pneumonia due to an exophytic lesion occluding the lumen of the main or lobar bronchi [21].

All the counting and measurement values took the average of the two radiologists. The differences were between two readers in the imaging interpretation of the presence of air bronchogram $(1 / 156,0.6 \%)$, vessel convergence sign $(3 / 156,1.9 \%)$, and pneumonia-like consolidation $(1 / 156$,
$0.6 \%$ ). Final conclusions regarding the CT examination findings were reached in consensus.

\section{Statistical analysis}

All statistical analyses were performed using SPSS (version 21.0; SPSS Inc., IBM Co., Chicago, IL, USA). Categorical variables or continuous variables were analyzed by chi-squared test, Fisher's exact test, ANOVA or Welch's ANOVA, as appropriate. All reported $P$ values were twosided, and a $P$ value $<0.05$ was considered as statistically significant. In intergroup chi-squared tests, the Bonferroni correction was used to counteract the problem of multiple comparisons; the $p$ value was 0.01 .

When the mono-factor analysis was complete, multivariable regression analysis was performed, after analysis of the multi-collinearity. A prediction tool for EGFR mutation was devised from the principal component analysis, which took the data to draw receiver operating characteristic (ROC) curves and calculate the available area under the curve (AUC). The Youden index was used to calculate the optimum cut-off.

\section{Results}

\section{Patient characteristics}

A total of 156 patients with adenocarcinomas were included in the analysis. One hundred patients harbored an EGFR mutation-exon 21 missense in 47 patients $(47.0 \%)$, exon 19 deletion in 44 (44.0\%), exon 20 insertion in $6(6.0 \%)$ and exon 18 deletion or missense mutation in 3 patients $(3.0 \%)$. One hundred and twenty-one patients had lung resection and 35 had open lung biopsy. EGFR mutation was seen more frequently in female patients $(57 / 100, P<0.001)$, non-smokers (78/100, $P<0.001)$, and normal and less severe emphysema patients $(95 / 100, P=0.016)$. There were no differences in age or tumor stage between wild-type and subtype of EGFRmutant lung adenocarcinomas (Table 1). After comparing the wild-type and each subtype of the mutation groups, the proportion of females and non-smokers in the exon 19 deletion and exon 21 missense groups was higher than in the wild-type group $(P<0.01)$. Severe emphysema was higher in the wild-type group than in the exon 19 deletion group $(P<0.01)$ (Table 2).

\section{CT imaging evaluation}

The Erel in the exon 18 deletion or missense mutation groups was lower than in the 19 deletion and wild-type groups $(P<0.05)$ (Tables 2, 3). The initial tumor size was measured in both the mediastinal window and the lung window. There 
Table 1 Clinical characteristics of wild-type and mutation subtypes
Table 2 Comprehensive tables with statistically significant differences

\begin{tabular}{|c|c|c|c|c|c|c|c|}
\hline \multirow[t]{2}{*}{ Characteristics } & \multirow[t]{2}{*}{ Total } & \multirow[t]{2}{*}{ Wild-type } & \multicolumn{4}{|c|}{ Mutation } & \multirow[t]{2}{*}{$P$} \\
\hline & & & 18 & 19 & 20 & 21 & \\
\hline Age (years) & & $61.482(41-80)$ & $\begin{array}{c}56.000 \\
(52- \\
62)\end{array}$ & $\begin{array}{c}60.136 \\
(32- \\
82)\end{array}$ & $\begin{array}{c}58.167 \\
(46- \\
76)\end{array}$ & $63.128(38-77)$ & 0.309 \\
\hline Gender & & & & & & & 0.000 \\
\hline Male & 90 & 47 & 2 & 17 & 3 & 21 & \\
\hline Female & 66 & 9 & 1 & 27 & 3 & 26 & \\
\hline Smoking history & & & & & & & 0.000 \\
\hline Smoker & 65 & 43 & 2 & 8 & 3 & 9 & \\
\hline Non-smoker & 91 & 13 & 1 & 36 & 3 & 38 & \\
\hline Stage & & & & & & & 0.639 \\
\hline IA & 37 & 11 & 1 & 11 & 2 & 12 & \\
\hline IB & 31 & 8 & 1 & 7 & 0 & 15 & \\
\hline IIA & 8 & 5 & 0 & 2 & 1 & 0 & \\
\hline IIB & 15 & 8 & 0 & 4 & 0 & 3 & \\
\hline IIIA & 33 & 14 & 1 & 9 & 2 & 7 & \\
\hline IIIB & 4 & 2 & 0 & 2 & 0 & 0 & \\
\hline IV & 28 & 8 & 0 & 9 & 1 & 10 & \\
\hline Emphysema & & & & & & & 0.016 \\
\hline Normal and score $<8$ & 140 & 45 & 2 & 43 & 5 & 45 & \\
\hline Score $\geq 8$ & 16 & 11 & 1 & 1 & 1 & 2 & \\
\hline
\end{tabular}

\begin{tabular}{|c|c|c|c|c|c|c|}
\hline \multirow[t]{2}{*}{ Characteristics } & \multirow[t]{2}{*}{ Wild-type } & \multicolumn{4}{|c|}{ Mutation } & \multirow[t]{2}{*}{$P$} \\
\hline & & 18 & 19 & 20 & 21 & \\
\hline Gender & 0 & & 0 & & & 0.000 \\
\hline Smoking history & 0 & & 0 & & & 0.000 \\
\hline Emphysema & 0 & & 0 & & & 0.008 \\
\hline MaxD mediastinal & 0 & & 0 & & & $<0.05$ \\
\hline TDR & 0 & & 0 & & & $<0.05$ \\
\hline GGO & 0 & & 0 & & & $<0.05$ \\
\hline Gender & 0 & & & & 0 & 0.000 \\
\hline Smoking history & 0 & & & & 0 & 0.000 \\
\hline MaxD mediastinal & 0 & & & & 0 & $<0.05$ \\
\hline MinD mediastinal & 0 & & & & 0 & 0.000 \\
\hline Erel & 0 & 0 & & & & $<0.05$ \\
\hline Erel & & 0 & 0 & & & $<0.05$ \\
\hline
\end{tabular}

were statistical differences between the wild-type group and EGFR mutation group in $\mathrm{MaxD}_{\text {mediastinal }}$ and $\mathrm{MinD}_{\text {mediastinal. }}$. The MaxD $\mathrm{D}_{\text {mediastinal }}$ in the wild-type group was longer than in the exon 19 deletion and exon 21 missense groups, and the $\mathrm{MinD}_{\text {mediastinal }}$ in the wild-type group was longer than in the exon 21 missense group, with a significant difference $(P<0.05)$ (Tables 2,3$)$. The TDR in the exon 19 deletion group was higher than in the wild-type group. GGO appeared to be more common in exon 19 deletion patients
$(P=0.010)$. According to the statistics, there were no significant differences between the groups with regard to the location of the lesions, attenuation, pneumonia-type, shape, boundary, lobulation, presence of spiculation, calcification, cavitation, air bronchogram, vascular convergence sign, pleural indentation, pericardial effusion, and pleural effusion (Table 3). 
Table 3 The CT features of wild-type and mutation subtypes

\begin{tabular}{|c|c|c|c|c|c|c|c|}
\hline \multirow[t]{2}{*}{ Characteristics } & \multirow[t]{2}{*}{ Total } & \multirow[t]{2}{*}{ Wild-type } & \multicolumn{4}{|l|}{ Mutation } & \multirow[t]{2}{*}{$P$} \\
\hline & & & 18 & 19 & 20 & 21 & \\
\hline \multicolumn{8}{|l|}{ Density (HU) } \\
\hline Non-enhanced & & $34.089 \pm 9.449$ & $34.333 \pm 6.351$ & $33.136 \pm 11.235$ & $37.667 \pm 8.824$ & $31.936 \pm 10.182$ & 0.675 \\
\hline Enhanced & & $60.946 \pm 13.770$ & $51.000 \pm 8.660$ & $60.250 \pm 14.379$ & $58.833 \pm 9.621$ & $57.553 \pm 14.359$ & 0.599 \\
\hline Erel & & $0.180 \pm 0.079$ & $0.126 \pm 0.013$ & $0.197 \pm 0.099$ & $0.166 \pm 0.086$ & $0.160 \pm 0.065$ & 0.002 \\
\hline \multicolumn{8}{|l|}{ Size $(\mathrm{mm})$} \\
\hline MaxD mediastinal & & $39.125(6-109)$ & $23.000(11-30)$ & $29.727(6-75)$ & $26.000(14-45)$ & $29.894(3-88)$ & 0.032 \\
\hline MinD mediastinal & & $31.518(5-93)$ & $21.000(11-26)$ & $23.864(4-52)$ & $22.333(10-42)$ & $22.809(2-50)$ & 0.160 \\
\hline MaxD lung & & $40.714(7-139)$ & $24.333(15-30)$ & 34.409 (13-92) & $31.167(15-45)$ & $33.574(11-101)$ & 0.271 \\
\hline MinD lung & & $32.875(6-99)$ & $22.333(15-26)$ & $27.273(11-61)$ & $28.000(12-46)$ & $26.250(9-52)$ & 0.311 \\
\hline TDR & & $0.071(0-0.443)$ & $0.154(0-0.462)$ & $0.206(0-0.926)$ & $0.267(0-0.701)$ & $0.197(0-0.962)$ & 0.042 \\
\hline Lobulation & & $4.589 \pm 3.431$ & $2.333 \pm 2.082$ & $4.341 \pm 2.745$ & $6.167 \pm 1.835$ & $4.745 \pm 3.473$ & 0.509 \\
\hline Homogeneity after contrast & & & & & & & 0.125 \\
\hline Heterogeneous & 65 & 30 & 0 & 16 & 3 & 16 & \\
\hline Homogenous & 91 & 26 & 3 & 28 & 3 & 31 & \\
\hline GGO & & & & & & & 0.034 \\
\hline Present & 36 & 5 & 1 & 15 & 2 & 13 & \\
\hline Absent & 120 & 51 & 2 & 29 & 4 & 34 & \\
\hline Pericardial effusion & & & & & & & 0.897 \\
\hline Present & 5 & 1 & 0 & 2 & 0 & 2 & \\
\hline Absent & 151 & 55 & 3 & 42 & 6 & 45 & \\
\hline Location & & & & & & & 0.164 \\
\hline Inferior lobe of left lung & 34 & 17 & 0 & 8 & 0 & 9 & \\
\hline Superior lobe of left lung & 29 & 8 & 1 & 9 & 2 & 9 & \\
\hline Middle lobe of right lung & 10 & 2 & 0 & 0 & 1 & 7 & \\
\hline Inferior lobe of right lung & 52 & 21 & 1 & 13 & 2 & 15 & \\
\hline Superior lobe of right lung & 31 & 8 & 1 & 14 & 1 & 7 & \\
\hline Pneumonia-type & & & & & & & 0.415 \\
\hline Pneumonia-type & 5 & 0 & 0 & 2 & 0 & 3 & \\
\hline Non-pneumonia-type & 151 & 56 & 3 & 42 & 6 & 44 & \\
\hline Shape & & & & & & & 0.400 \\
\hline Round or oval & 110 & 40 & 3 & 34 & 4 & 29 & \\
\hline Irregular & 46 & 16 & 0 & 10 & 2 & 18 & \\
\hline Spiculation & & & & & & & 0.899 \\
\hline Present & 108 & 37 & 2 & 30 & 5 & 34 & \\
\hline Absent & 48 & 19 & 1 & 14 & 1 & 13 & \\
\hline Boundary & & & & & & & 0.103 \\
\hline Clear & 146 & 52 & 3 & 38 & 6 & 47 & \\
\hline Obscure & 10 & 4 & 0 & 6 & 0 & 0 & \\
\hline Calcification & & & & & & & 0.435 \\
\hline Present & 9 & 5 & 0 & 2 & 1 & 1 & \\
\hline Absent & 147 & 51 & 3 & 42 & 5 & 46 & \\
\hline Cavitation & & & & & & & 0.960 \\
\hline Present & 27 & 9 & 1 & 8 & 1 & 8 & \\
\hline Absent & 129 & 47 & 2 & 36 & 5 & 39 & \\
\hline Air bronchogram & & & & & & & 0.206 \\
\hline Present & 38 & 10 & 0 & 7 & 3 & 18 & \\
\hline Absent & 118 & 46 & 3 & 37 & 3 & 29 & \\
\hline Vascular convergence sign & & & & & & & 0.116 \\
\hline
\end{tabular}


Table 3 (continued)

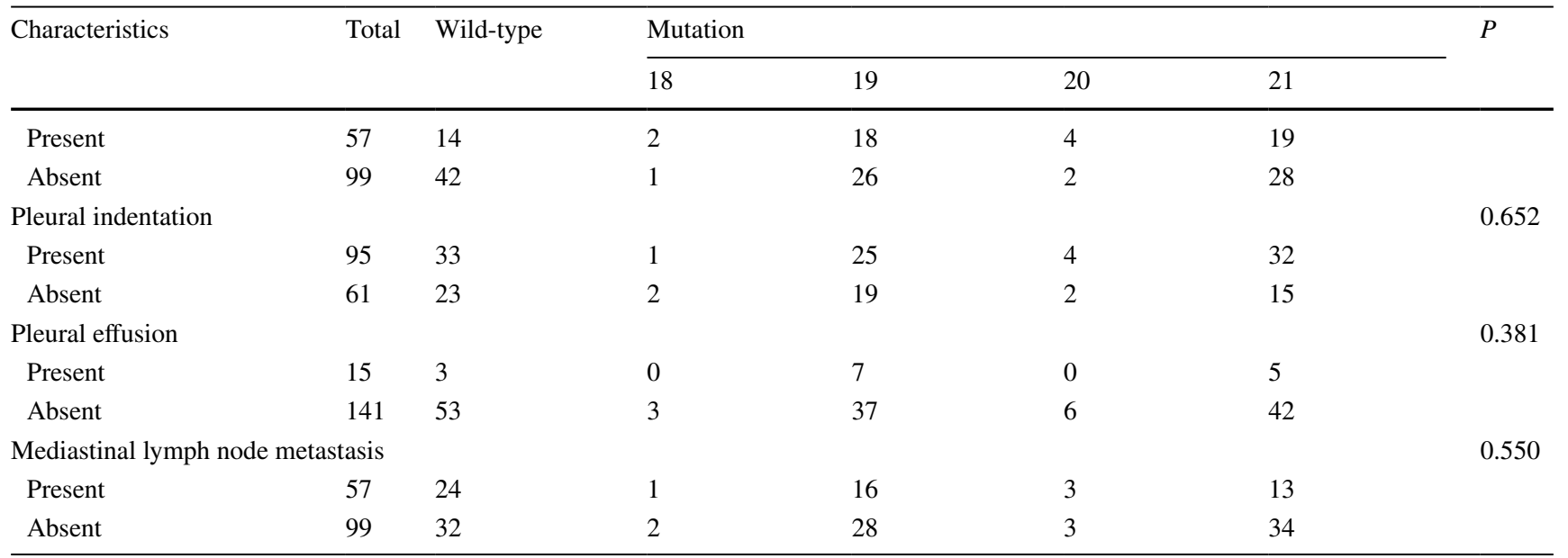

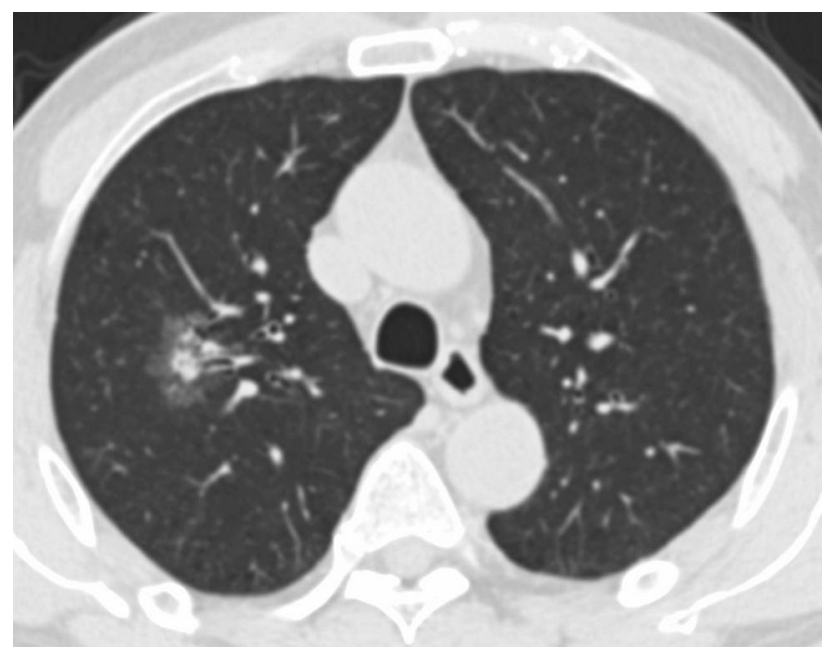

Fig. 1 F, 42Y, exon 19 deletion positive, the lesion located in the superior right lobe was a mixed GGO

\section{Prediction model of EGFR mutation from CT findings}

After the mono-factor analysis, we found gender, smoking history, emphysema, MaxD $\mathrm{D}_{\text {mediastinal }}$, TDR and GGO showed statistical differences between the wild-type group and the exon 19 deletion group (Fig. 1). We performed a collinearity diagnosis of the 6 factors (the condition index was 12.970). We then extracted the main factors by principal component analysis, and the prediction score was calculated by the product sum of the eigenvector and standardized CT and clinical feature variables: $0.305 \times$ gender $+0.254 \times$ smoke history $+0.198 \times$ MaxDmediastinal + TDR $\times 0.254+0.280 \times$ GGO + $0.095 \times$ emphysema. The AUC was 0.670, the Youden index was 0.359, the cut-off value was -0.057 , and the sensitivity and specificity for predicting exon 19 deletion were 59.09 and $76.79 \%$, respectively (Fig. 2).

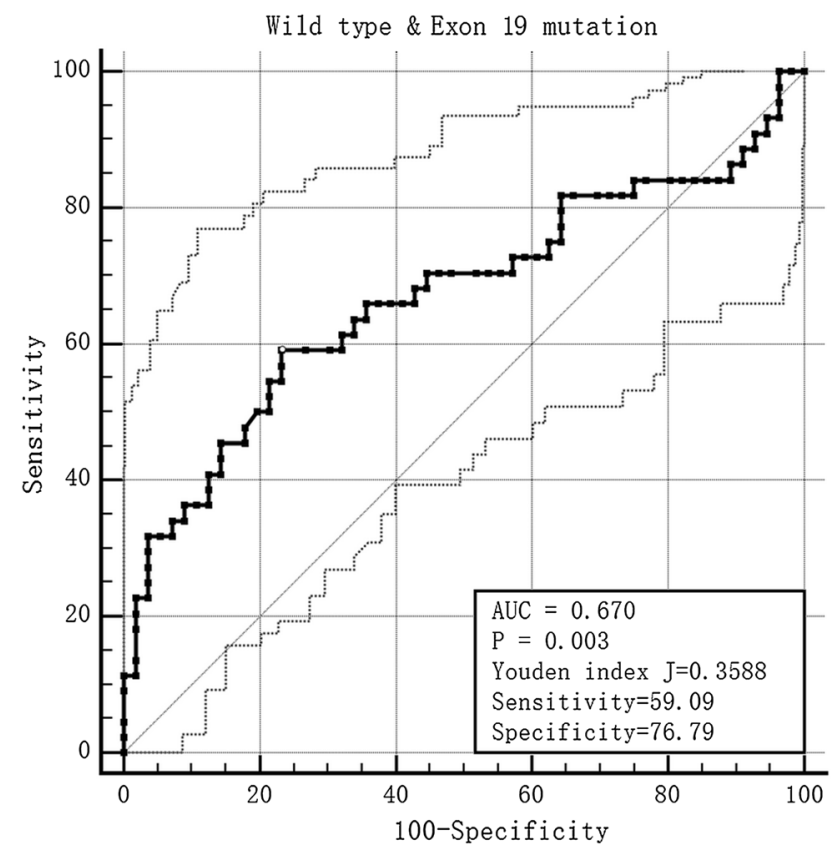

Fig. 2 The ROC of the prediction score in the wild-type and exon 19 mutation groups

The condition index of collinearity diagnosis in the wildtype and exon 21 missense groups was 18.074, meaning that there was multiple co-linear relationships between the parameters. We also used principal component analysis to solve the problem. The prediction score was calculated by the product sum of the eigenvector and standardized CT and clinical feature variables: $0.354 \times$ gender $+0.291 \times$ smoke history $+0.410 \times \mathrm{MaxD}_{\text {mediastinal }}+0.408 \times \mathrm{MinD}^{\text {mediastinal }}$. The AUC was 0.791 , the Youden index was 0.509 , the cutoff value was -0.231 , and the sensitivity and specificity for predicting exon 21 missense were 72.34 and $78.57 \%$, respectively (Fig. 3). 


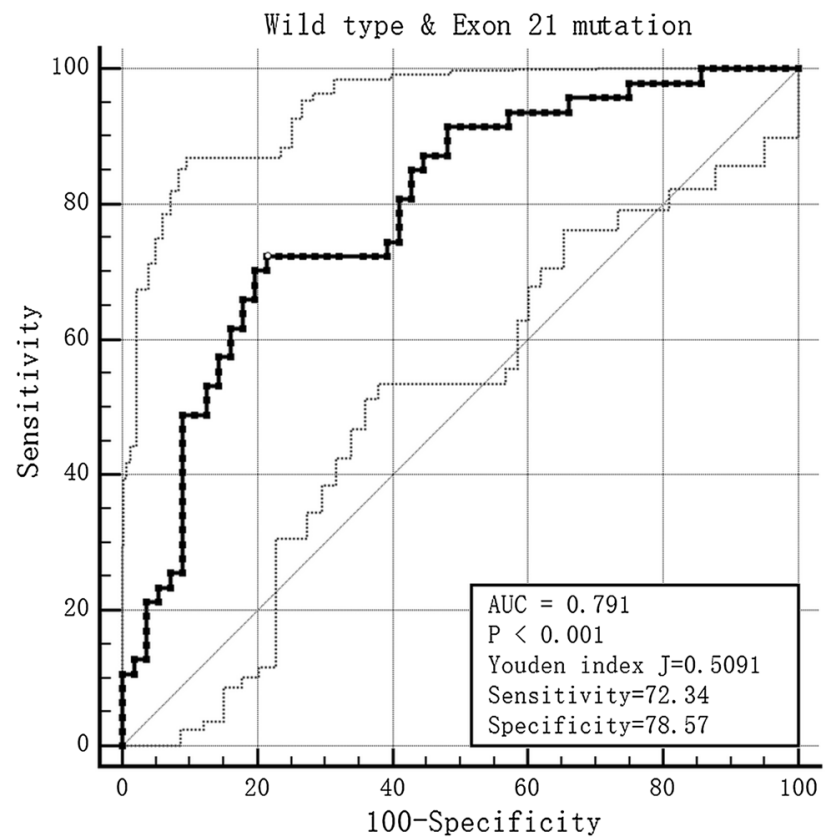

Fig. 3 The ROC of the prediction score in the wild-type and exon 21 mutation groups

After regression analysis with wild-type and exon 18 deletion or missense groups in Erel, the $P$ value was 0.238 , and in the wild-type and exon 18 deletion or missense groups it was 0.242 .

\section{Discussion}

There were two main parts to our study. In the first part, we evaluated the differences in various clinical features and CT features between different genotypes in lung adenocarcinoma. The results showed that exon 19 deletion EGFR mutation was associated with gender, smoking history, the MaxD $\mathrm{D}_{\text {mediastinal }}$, TDR, GGO and emphysema grade, and the exon 21 missense mutation was associated with gender, smoking history, and the $\mathrm{MaxD}_{\text {mediastinal }}$ and $\mathrm{MinD}_{\text {mediastinal }}$. There were statistical differences between the wild-type and exon 18 deletion or missense groups in Erel, and also between the exon 18 deletion or missense and exon 19 deletion groups. In the second part, we explored the predictive ability of these factors by multiple logistic regression analysis and principal component analysis. The sensitivity and specificity of the prediction score that we devised for exon 19 deletion EGFR mutation were 59.09 and $76.79 \%$, respectively, and for exon 21 missense mutation were 72.34 and $78.57 \%$, respectively. However, the Erel had no prediction value for the diagnosis.

The purposes of the scoring system are as follows. First, the EGFR tyrosine kinase inhibitors have a diverse curative effect in different subtypes of the EGFR mutation, so the scoring system may be useful to predict the curative effect in a non-invasive way. Second, it can be used in those patients who are not suitable for surgery or other invasive operations; this scoring system may be useful in helping to decide the EGFR mutation status. Third, it may avoid unnecessary false-negative results, and help to decide whether the repeat biopsy or surgery is worth performing for patients with inconsistent genetic testing and clinical features.

Our study focused on a population of East Asian origin. Compared to a Western population, a population of East Asian origin with NSCLC had higher rates of EGFR mutations [22, 23]. Our study demonstrated that EGFR mutation lesions were significantly higher in females and in nonsmokers. Indeed, we identified EGFR mutations in $64.10 \%$ of all cases; female patients comprised $57 \%$ in the mutation group, which was consistent with previous studies [20, 24-26], especially in the subtypes of the exon 19 deletion and exon 21 missense groups.

Besides the demographic characteristics, our study had an interesting feature in that there was a relationship between the Goddard scoring system and exon 19 deletion. According to the modified Goddard scoring system [16], it was considered to be a representative value of the severity of emphysema in each person, and was very convenient to apply. In our study, a minimum score of 0 to a maximum score of 8 was considered a representative value of the mild type of emphysema in the lungs; when the score was $>8$, it was considered as severe emphysema. The results showed the incidence of exon 19 deletion was higher in the mild type $(97.73 \%)$ than in the wild-type (80.35\%). Furthermore, the reason for this difference may be correlated with the cause of the emphysema, as smoking is a major risk factor for the development and progression of chronic obstructive pulmonary disease and lung cancer [16, 27, 28], and the EGFR mutation lesions were significantly higher in females and in non-smokers. We propose that the present results may be valuable in clinical practice, because to define emphysema using the Goddard scoring system is very easy to apply, requires little clinical testing, and can provide complementary information for diagnosing.

Most of the CT features had no significant statistical differences. We demonstrated that $\mathrm{MaxD}_{\text {mediastinal }}$, TDR, and the presence of GGO showed statistical differences between the exon 19 deletion and wild-type groups, and $\mathrm{MaxD}_{\text {mediastinal }}$ and $\mathrm{MinD}_{\text {mediastina }}$ showed statistical differences between the exon 21 missense and wild-type groups. The relationship between GGO and EGFR has been frequently studied [6, 20, 24-26]. GGO appeared to be more common in EGFR mutation patients than in wild-type patients. A few articles have reported that the presence of GGO was more significant in exon 21 missense mutation, and no difference was seen in 
exon 19 deletion and wild-type patients [26]; however, some have reported differences in wild-type and both exon mutations [25]. These conclusions were somewhat out of line with our observations, although it also indicates the diagnostic value of GGO. These differences might be due to the study population and the diagnostic procedures.

TDR has been proposed as a new radiologic variable. It is calculated from the tumor shadow on both the lung and mediastinal windows on CT images, to represent the proportion of the GGO area in the entire lesion [29]. Previous research used semi-automated nodule assessment software and showed that there was no significant difference regarding TDR between the EGFR mutation type and the wild-type [30]. One reason for the differences might be the fact that our study focused on the differences between the wild-type and the EGFR mutation subtype, as well as using a different assessment method. However, to our knowledge, this feature has not been previously reported. It can be used to quantify the shape of the tumor but further studies are needed to test and verify our results.

We measured both the maximum and minimum diameter of the mediastinal window and the lung window. The results showed the $\mathrm{MaxD}_{\text {mediastinal }}$ of the wild type was longer than the exon 19-deletion group, and in the wildtype and exon 21 missense groups, both the $\mathrm{MaxD}_{\text {mediastinal }}$ and $\mathrm{MinD}_{\text {mediastinal }}$ of the wild-type were longer. Previous research also focused on this issue; some studies measured the average diameter of the lesion, and some only measured the diameter in the lung window. Lee et al. demonstrated that in the early stage of lung adenocarcinoma, the diameter of the EGFR mutation tumor was longer than the wild-type; however, the measurement methods were not specified [31]. Usuda et al. measured the maximum tumor diameter in chest $\mathrm{CT}$ and the result showed that the diameter in the wild-type group was longer than in the mutation group [32]. Liu et al. [20] measured both the maximum and minimum tumor diameter and also reached the same result as the study by Usuda et al. [20], which was partly in agreement with our result. According to the literature, the criteria for diameter measurement vary widely. Whether or not they are associated with EGFR mutations is also controversial; they might be related to different races and different stages of lung adenocarcinoma. This deserves further study in the follow-up work.

Erel is a relatively standardized enhancement index. Only a few articles focus on this parameter and have shown no statistical significance [20]. Our study showed there were some statistical differences between the wild-type and exon 18 mutation groups, as well as between the exon 18 and 19 mutation groups; however, after logistic regression, there was no statistical difference between the groups. This suggests that Erel has little predictive value in the diagnosis of EGFR mutations. There might be two reasons for this. One reason is that EGFR mutations may have nothing to do with Erel, and the other is that the results might be due to the small sample size; the exon 18 mutation group had only 3 patients which will have had a bearing on the credibility of the results. We may need to collect more relevant cases in the follow-up work to obtain more accurate results.

In our study, we quantitatively measured some of the CT parameters, such as diameter, TDR, Erel, emphysema, and found that gender, smoking history, emphysema, diameter and TDR had some predictive value in wild-type and different EGFR mutation subtypes. We then established a prediction model to help predict whether there is a mutation. Screening some parameters such as the value of Erel has laid the foundation for further research. We also found that Erel had some statistical significance, but because of the small amount of data, further research is needed.

As a retrospective cohort study, our study had several limitations. We used two different CT scanners, so the scanning parameters were not same and this might cause bias to the results. As this study was performed in a single large medical center, there may have been bias in the selection of patients; the sample size was not relatively large and not predetermined in dimension with a power analysis.

To our knowledge, although many researchers have focused on the relationship between the genotype, this study is the first to present and discuss some new findings among EGFR mutation subtype and wild-type lung adenocarcinomas. As well as gender, smoking history and GGO, adenocarcinomas with EGFR mutations were significantly associated with emphysema, TDR, and the diameter in mediastinal window. As some EGFR mutation subtypes might be predicted by these features, the scoring system might be valuable for clinical diagnosis.

\section{Compliance with ethical standards}

Conflict of interest The authors have no conflict of interest to declare.

Open Access This article is distributed under the terms of the Creative Commons Attribution 4.0 International License (http://creativecommons.org/licenses/by/4.0/), which permits unrestricted use, distribution, and reproduction in any medium, provided you give appropriate credit to the original author(s) and the source, provide a link to the Creative Commons license, and indicate if changes were made.

\section{References}

1. Siegel RL, Miller KD, Jemal A (2016) Cancer statistics, 2016. CA Cancer J Clin 66(1):7-30. doi:10.3322/caac. 21332

2. Chen W, Zheng R, Baade PD et al (2016) Cancer statistics in China, 2015. CA Cancer J Clin 66(2):115-132. doi:10.3322/ caac. 21338

3. Ozkan E, West A, Dedelow JA et al (2015) CT Gray-level texture analysis as a quantitative imaging biomarker of epidermal growth 
factor receptor mutation status in adenocarcinoma of the lung. Am J Roentgenol 205(5):1016-1025. doi:10.2214/AJR.14.14147

4. Nishino M, Hatabu H, Johnson BE et al (2014) State of the art: response assessment in lung cancer in the era of genomic medicine. Radiology 271(1):6-27. doi:10.1148/radiol.14122524

5. Nishino M, Jackman DM, Hatabu $\mathrm{H}$ et al (2011) Imaging of lung cancer in the era of molecular medicine. Acad Radiol 18(4):424 436. doi:10.1016/j.acra.2010.10.020

6. Hsu JS, Huang MS, Chen CY et al (2014) Correlation between EGFR mutation status and computed tomography features in patients with advanced pulmonary adenocarcinoma. J Thorac Imaging 29(6):357-363. doi:10.1097/RTI.0000000000000116

7. Antonicelli A, Cafarotti S, Indini A et al (2013) EGFR-targeted therapy for non-small cell lung cancer: focus on EGFR oncogenic mutation. Int J Med Sci 10(3):320-330. doi:10.7150/ijms.4609

8. Gazdar AF (2009) Personalized medicine and inhibition of EGFR signaling in lung cancer. N Engl J Med 361(10):1018-1020. doi:10.1056/NEJMe0905763

9. Maemondo M, Inoue A, Kobayashi K et al (2010) Gefitinib or chemotherapy for non-small-cell lung cancer with mutated EGFR. N Engl J Med 362(25):2380-2388. doi:10.1056/NEJMoa0909530

10. Wu JY, Wu SG, Yang CH et al (2008) Lung cancer with epidermal growth factor receptor exon 20 mutations is associated with poor gefitinib treatment response. Clin Cancer Res 14(15):4877-4882. doi:10.1158/1078-0432.CCR-07-5123

11. He M, Capelletti M, Nafa K et al (2012) EGFR exon 19 insertions: a new family of sensitizing EGFR mutations in lung adenocarcinoma. Clin Cancer Res 18(6):1790-1797. doi:10.1158/1078-0432. CCR-11-2361

12. Shigematsu H, Lin L, Takahashi $T$ et al (2005) Clinical and biological features associated with epidermal growth factor receptor gene mutations in lung cancers. J Natl Cancer Inst 97(5):339-346. doi:10.1093/jnci/dji055

13. Wu JY, Yu CJ, Chang YC et al (2011) Effectiveness of tyrosine kinase inhibitors on "uncommon" epidermal growth factor receptor mutations of unknown clinical significance in nonsmall cell lung cancer. Clin Cancer Res 17(11):3812-3821. doi:10.1158/1078-0432.CCR-10-3408

14. Inukai M, Toyooka S, Ito S et al (2006) Presence of epidermal growth factor receptor gene T790M mutation as a minor clone in non-small cell lung cancer. Cancer Res 66(16):7854-7858. doi:10.1158/0008-5472.CAN-06-1951

15. Lee JK, Hahn S, Kim DW et al (2014) Epidermal growth factor receptor tyrosine kinase inhibitors vs conventional chemotherapy in non-small cell lung cancer harboring wild-type epidermal growth factor receptor: a meta-analysis. JAMA 311(14):1430 1437. doi:10.1001/jama.2014.3314

16. Kitaguchi Y, Fujimoto K, Kubo K et al (2006) Characteristics of COPD phenotypes classified according to the findings of HRCT. Respir Med 100(10):1742-1752. doi:10.1016/j.rmed.2006.02.003

17. Edge SB, Compton CC (2010) The American Joint Committee on Cancer: the 7th edition of the AJCC cancer staging manual and the future of TNM. Ann Surg Oncol 17(6):1471-1474. doi:10.1245/ s10434-010-0985-4

18. Travis WD, Brambilla E, Nicholson AG et al (2015) The 2015 World Health Organization Classification of Lung Tumors: impact of genetic, clinical and radiologic advances since the 2004 classification. J Thorac Oncol 10(9):1243-1260. doi:10.1097/ JTO.0000000000000630

19. Nakada T, Okumura S, Kuroda $\mathrm{H}$ et al (2015) Imaging characteristics in alk fusion-positive lung adenocarcinomas by using HRCT. Ann Thorac Cardiovasc Surg 21(2):102-108. doi:10.5761/atcs. oa.14-00093

20. Liu Y, Kim J, Qu F et al (2016) CT features associated with epidermal growth factor receptor mutation status in patients with lung adenocarcinoma. Radiology 280(1):271-280. doi:10.1148/ radiol.2016151455

21. Liu J, Shen J, Yang C et al (2015) High incidence of EGFR mutations in pneumonic-type non-small cell lung cancer. Medicine (Baltimore) 94(8):e540. doi:10.1097/MD.0000000000000540

22. Dearden S, Stevens J, Wu YL et al (2013) Mutation incidence and coincidence in non small-cell lung cancer: meta-analyses by ethnicity and histology (mutMap). Ann Oncol 24(9):2371-2376. doi:10.1093/annonc/mdt205

23. El-Telbany A, Ma PC (2012) Cancer genes in lung cancer: racial disparities: are there any? Genes Cancer 3(7-8):467-480. doi:10.1177/1947601912465177

24. Ha SY, Choi SJ, Cho JH et al (2015) Lung cancer in neversmoker Asian females is driven by oncogenic mutations, most often involving EGFR. Oncotarget 6(7):5465-5474. doi:10.18632/ oncotarget. 2925

25. Hasegawa M, Sakai F, Ishikawa R et al (2016) CT features of epidermal growth factor receptor-mutated adenocarcinoma of the lung: comparison with nonmutated adenocarcinoma. J Thorac Oncol 11(6):819-826. doi:10.1016/j.jtho.2016.02.010

26. Lee HJ, Kim YT, Kang CH et al (2013) Epidermal growth factor receptor mutation in lung adenocarcinomas: relationship with CT characteristics and histologic subtypes. Radiology 268(1):254264. doi:10.1148/radiol.13112553

27. Postma DS, Kerkhof M, Boezen HM et al (2011) Asthma and chronic obstructive pulmonary disease: common genes, common environments? Am J Respir Crit Care Med 183(12):1588-1594. doi:10.1164/rccm.201011-1796PP

28. Lundback B, Lindberg A, Lindstrom M et al (2003) Not 15 but $50 \%$ of smokers develop COPD? - Report from the obstructive lung disease in Northern Sweden Studies. Respir Med 97(2): $115-122$

29. Okada M, Nishio W, Sakamoto T et al (2003) Discrepancy of computed tomographic image between lung and mediastinal windows as a prognostic implication in small lung adenocarcinoma. Ann Thorac Surg 76(6): 1828-1832 (discussion 1832)

30. Zhou JY, Zheng J, Yu ZF et al (2015) Comparative analysis of clinicoradiologic characteristics of lung adenocarcinomas with ALK rearrangements or EGFR mutations. Eur Radiol 25(5):12571266. doi:10.1007/s00330-014-3516-Z

31. Lee Y, Lee HJ, Kim YT et al (2013) Imaging characteristics of stage I non-small cell lung cancer on CT and FDG-PET: relationship with epidermal growth factor receptor protein expression status and survival. Korean J Radiol 14(2):375-383. doi:10.3348/ kjr.2013.14.2.375

32. Usuda K, Sagawa M, Motono N et al (2014) Relationships between EGFR mutation status of lung cancer and preoperative factors are they predictive? Asian Pac J Cancer Prev 15(2):657-662 